\title{
ENHANCEMENT OF WEAR RESISTANCE OF D-3 PIERCING PUNCHES BY APPLYING CRYOGENIC TREATMENT: FIELD AND LABORATORY INVESTIGATIONS
}

\begin{abstract}
Summary
Due to excessive shearing and load, punches wear faster than dies in press tools used for cutting operations. The shape and size of components produced by worn punches pose challenges; hence efforts are being taken by industries to extend the punch life and to reduce the cost and time of replacement of punches. The paper reports on an attempt made to investigate effects of cryogenic treatment and tempering cycles on wear life enhancement of D-3 tool steel specimens and punches. Four differently treated groups of D-3 piercing punches and pins were tested in the manufacture of AISI 1055 medium carbon steel chain parts and pinon-disk wear tests were carried out. The punch wear was assessed by capturing images of the punch tips using a machine vision system after manufacturing several chain components and the image processing technique was used in assessing the wear. Scanning electron microscopy (SEM) and optical microscopy (OM) images of the D-3 specimens were also taken to study effects of the cryogenic treatment. The results show improved wear resistance of the cryogenically treated punches compared to the conventionally heat-treated punches.
\end{abstract}

Key words: $\quad$ cryogenic treatment, piercing, punch wear, image processing, machine vision

\section{Introduction}

Globally, metal forming industries, in which press tools play an important role, are growing rapidly. Cutting and forming press tools are widely used in mass production in various sectors. Press tools are mounted on presses to perform required operations. These tools produce components from either coil stock or unit stock sheet metal. Blanking and piercing tools, in which the combined action of punch and die surface cuts and removes the component of a required size and shape by shearing action, are popular among the press tools used for cutting operations. Blanking and piercing look similar in operation but they differ in their applications. While performing blanking and piercing operations some amount of material is cut out and removed from the stock material, which is the component in blanking and scrap in piercing. Due to severe market competition, industries are engaged in research and development activities to increase the tool life and to reduce machining cost and lead time. 
The quality and dimensions of workpieces produced by the sheet metal cutting operations blanking and piercing depend on factors such as tool design, material properties, operating conditions, and especially punch wear [1-3]. Punches and dies are exposed to heavy loads and wear occurs due to adhesion and abrasion on cutting edges during the stamping of parts, especially from heat-treated steel blanks. Extreme punch wear causes defective part geometry and increases burr size, which leads to rejection of parts $[4,5]$. As part defects are related to the wear of cutting elements, mainly due to punch wear, [6, 7], monitoring of punch wear throughout the cutting process is very much needed. The contour of a worn punch [8-13] is widely used to decide whether the punch is suitable for further use.

Machine vision based systems are becoming popular solutions in tool condition monitoring. The noncontact measurement using image processing technology and machine vision systems can be effectively used for tool condition monitoring and includes the capability of enhanced automation in unmanned machines [14, 15].

The productivity of metal stamping can be increased by reducing wear and prolonging punch life $[10,16]$. Worn-out punches are to be replaced periodically, often at a significant economic cost $[17,18]$. Design changes, speciality materials, hard coatings, heat treatment practices and manufacturing methods are few areas where attempts are being made by industries to increase punch life, thereby increasing productivity and cost reduction $[19,20]$.

Cryogenic treatment is an additional process to the conventional heat treatment introduced between quenching and tempering. In this treatment the micro-structure of metals is modified by utilizing ultra-cold temperatures, which results in increased hardness, wear resistance and toughness of specimens. During the cryogenic treatment quenched steel specimens are cooled down to a liquid nitrogen temperature $\left(-196{ }^{\circ} \mathrm{C}\right)$ at a uniform cooling rate, soaked for a sufficient time period in liquid nitrogen and brought back to room temperature at a uniform rate [21]. As alloy tool steels have high carbon content, the transformation of austenite into martensite is completed below $0{ }^{\circ} \mathrm{C}$ only, which means that even after heat treatment austenite is still retained at room temperature. Cryogenic treatment transforms the retained austenite into martensite, which is effective in wear resistance improvement. Cryogenic treatment also promotes precipitation and uniform distribution of fine carbides in the matrix [22-24]. Laboratory investigations and shop floor experiments on cryogenically treated cutting tools for turning, milling cutters, drills and carbide tools have established performance enhancement of the tools [25-30].

However, more shop floor tests are needed to investigate the performance of cryogenically treated tool steels in press tool applications. In the present work, the effect of cryogenic treatment on punch wear was tested in the shop floor using cryogenically treated D3 piercing punches in the production of chain parts and a machine vision system with an image processing technique was used to monitor the punch wear periodically. Microstructural studies by means of optical microscopy (OM) and scanning electron microscopy (SEM) images in addition to pin-on-disk wear tests were also performed on the cryogenically treated D-3 steel pins. 
Field and Laboratory Investigations

\section{Experimental details}

\subsection{Material selection}

Commercial grade D-3 tool steel was chosen for piercing punches and pins to produce inner plates of industrial chains and for performing wear test. The composition of the chosen steel is (wt \%) $2.00 \%$ to $2.35 \% \mathrm{C}, 11.00 \%$ to $13.50 \% \mathrm{Cr}, 0.10 \%$ to $0.60 \% \mathrm{Mn}, 0.00 \%$ to $0.03 \% \mathrm{P}, 0.10 \%$ to $0.60 \% \mathrm{Si}$ and $1.00 \% \max \mathrm{V}$, which is in accordance with the AISI specifications for D-3 steel. The D-3 piercing punches were designed and manufactured as shown in Fig. 1 and they were used to punch holes of $11.47 \mathrm{~mm}$ in diameter from $3.60 \mathrm{~mm}$ thick AISI 1055 medium carbon steel blanks to produce inner plate components for industrial chains (Fig. 2). The chemical composition (wt \%) of the AISI 1055 steel blank material consists of $0.500 \%$ to $0.600 \% \mathrm{C}, 0.600 \%$ to $0.900 \% \mathrm{Mn}, 0.015 \% \mathrm{~S}$, and $0.020 \% \mathrm{P}$. The medium carbon steel blanks were in annealed condition with reduced hardness.

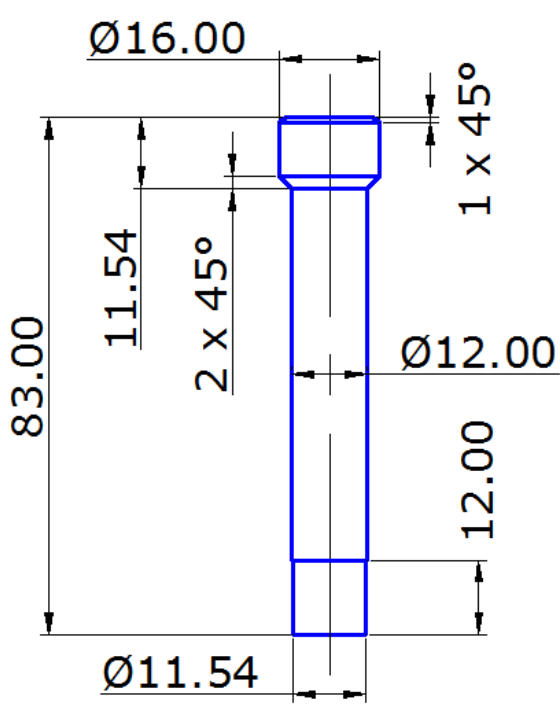

(a)

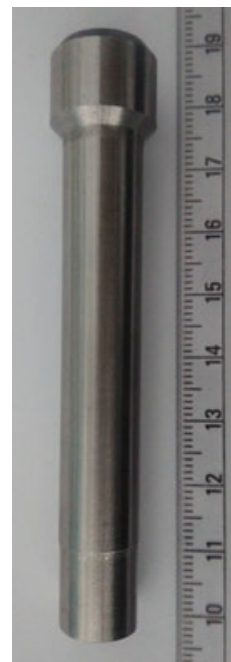

(b)

Fig. 1 D-3 piercing punch used for field experiments (b) and its dimensions (a)

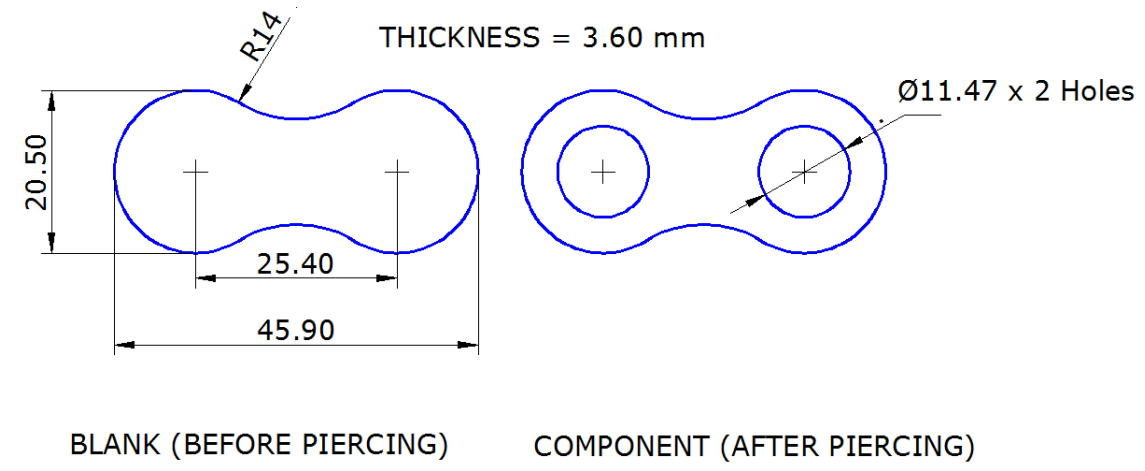

Fig. 2 Dimensions of AISI 1055 medium carbon steel blank used for field experiments and pierced component

\subsection{Treatments}

Four groups of D-3 steel piercing punches and pins were subjected to the treatment sequence as shown in Fig. 3. 


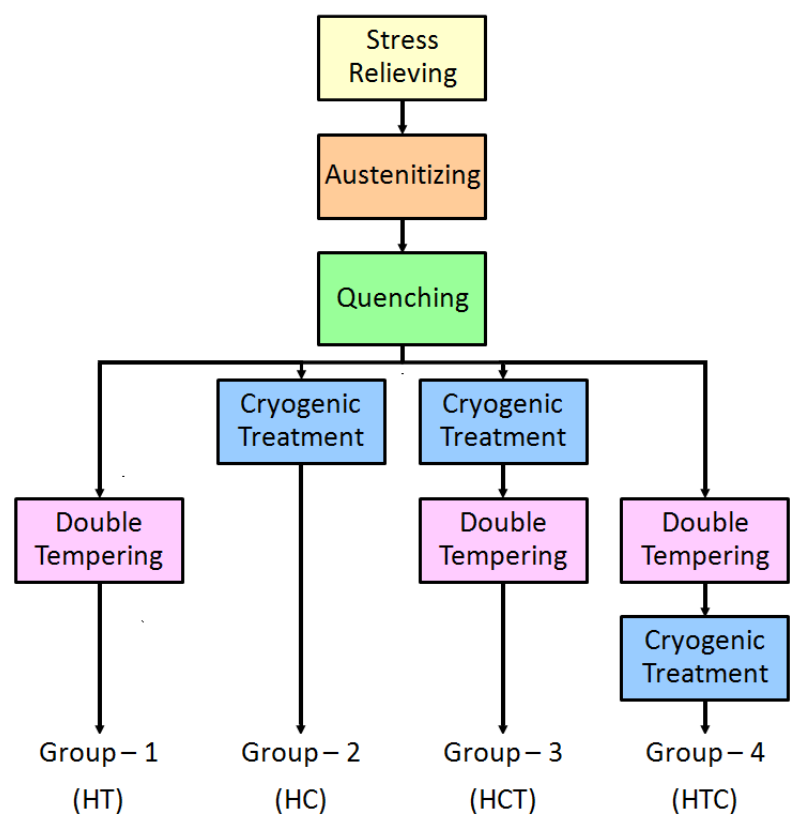

Fig. 3 Specimen treatment sequence

The specimens were first stress relieved at $600{ }^{\circ} \mathrm{C}$ by being soaked for 1 hour in an oil fired furnace followed by furnace cooling. They were then preheated to $200{ }^{\circ} \mathrm{C}$, held at the temperature for 30 minutes followed by their heating to $800^{\circ} \mathrm{C}$ and holding for 30 minutes for pre-austenitation in the oil fired furnace. Finally, austenitation was performed by heating the specimens to $950{ }^{\circ} \mathrm{C}$ and holding them at the temperature for 30 minutes. Then the specimens were oil quenched at $200{ }^{\circ} \mathrm{C}$ for 45 minutes followed by air-cooling to room temperature. These hardened specimens were divided into 4 groups, namely HT, HC, HCT and HTC. Subsequently, they were subjected to tempering and cryogenic processing. Tempering of the specimens was carried out in the oil fired furnace. After hardening, the specimens of the groups HT and HTC were immediately soaked at $200{ }^{\circ} \mathrm{C}$ for one hour, then air cooled to room temperature for first tempering. The air cooled specimens were tempered the second time by being soaked at the same temperature of $200{ }^{\circ} \mathrm{C}$ for one hour followed by air cooling to room temperature. The hardened specimens of the groups HC and HCT were cryogenically processed in a cryogenic chamber by bringing them to $-196^{\circ} \mathrm{C}$ from room temperature gradually in one hour and then they were soaked at $-196{ }^{\circ} \mathrm{C}$ for 10 hours and brought back to room temperature gradually in one hour. The specimens of the group HCT were double tempered, as mentioned earlier, immediately after the cryogenic processing. The specimens of the group $\mathrm{HC}$ were not tempered after the cryogenic processing. The specimens of the group HTC were cryogenically processed after double tempering by following the procedure as mentioned above. After these treatments all the specimens were machined to the final size.

\subsection{Hardness measurement}

The micro-hardness values of the carefully and properly prepared specimens after treatments were measured by using a Vickers microhardness tester for $0.2 \mathrm{~kg}$ load and a reliable value was obtained by carrying out the test several times. Equivalent HRC values were obtained and tabulated. 
Punches by Applying Cryogenic Treatment:

Field and Laboratory Investigations

\subsection{Microstructural examination}

For microstructural studies the samples were prepared from all the four groups of D-3 steel specimens after the treatment carried out according to the standard metallographic procedure. Viella's reagent composed of $1 \mathrm{gm}$ picric acid, $5 \mathrm{ml} \mathrm{HCl}$ and $100 \mathrm{ml}$ ethanol was used for revealing the microstructure i.e. the tempered martensite will appear better.

The microstructural analysis of the prepared D-3 specimens was carried out on images taken by a JEOL JSM-6390 Scanning Electron Microscope (SEM) at a magnification of 3500x and by a CARL ZEISS Optical Microscope at a magnification of 1000x.

\subsection{Piercing experiments}

In the production of inner plates of industrial chains the piercing operation was performed in a Godrej SC1-63 single crank straight side 63 ton press at a speed of 130 strokes/min. The piercing tool was operated at room temperature under a constant thin-film lubrication regime. The prepared punches were used on the provided blanks to pierce 11.47 $\mathrm{mm}$ diameter holes, as shown in Fig. 4, in the press. The punches fitted in the press for the purpose of conducting field experiments are shown in Fig. 5. To investigate wear progression as a function of the number of strokes, the punches were removed from the piercing tool after $7,000,14,000,21,000$ and 28,000 strokes. In each case, a new punch was used from every group of the prepared punch samples. The removed punches were used to assess the wear progression by the machine vision system and the image processing technique.
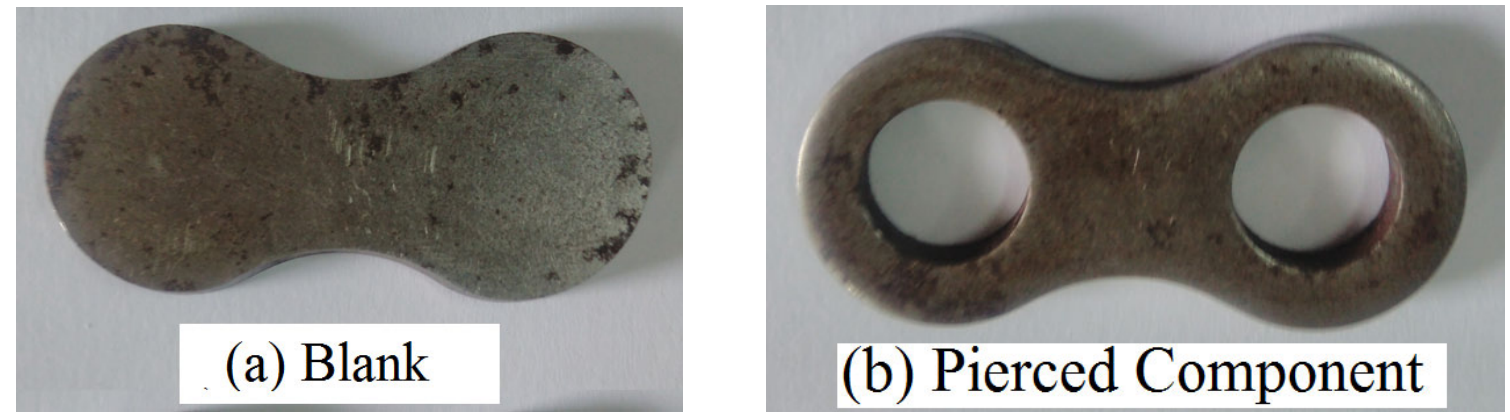

Fig. 4 AISI 1055 steel blank used for piercing and pierced component

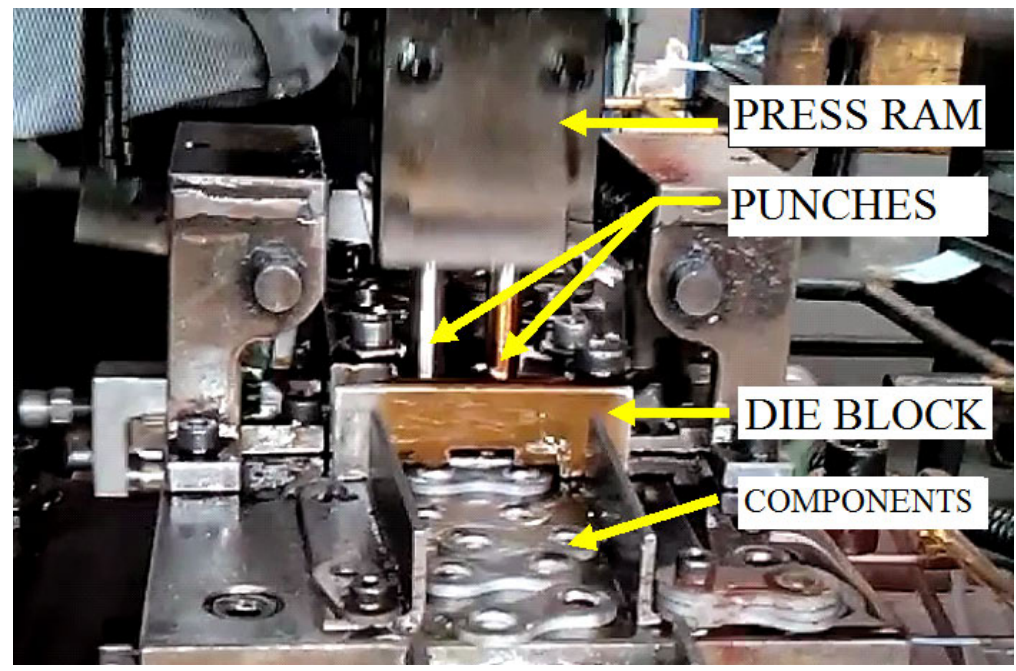

Fig. 5 Experimental setup in the press 


\subsection{Machine vision system}

The machine vision system for measuring the punch wear consists of a camera, a lens, an illumination source, a PC and suitable software for acquiring images from the punches removed from the piercing tool.

\subsubsection{Selection of camera and light source}

The setup acquires images via a Matrix Vision (mvBlueCOUGAR-X125AG) camera and Bi-Telecentric lenses (TC Series) with a circular field of view of $60 \mathrm{~mm}$ in diameter and high resolution (2448 $\times 2050)$, that are used to assess wear on the piercing punches by means of highly detailed information contained in the images. The Bi-Telecentric lenses ensure pure telecentricity. Hence, no magnification change occurs when the punches move away or towards the system. Proper illumination is the most important and critical aspect of obtaining a good image for further processing. Hence, an axial diffuse illuminator (6" x 6", DL085) was used because it is suitable for use on highly reflective objects and when there are shadows on the inspection area.

\subsubsection{Image acquisition procedure}

For acquiring images, the punch is mounted in a fixture. While the illumination is on, images are acquired using the software interface wxPropView. Positioning of the punches and the illumination settings are critical factors for the image quality as they have an exaggerated effect when punch wear is determined. The wear evolution has been determined from the images acquired by using the machine vision system (Fig. 6).

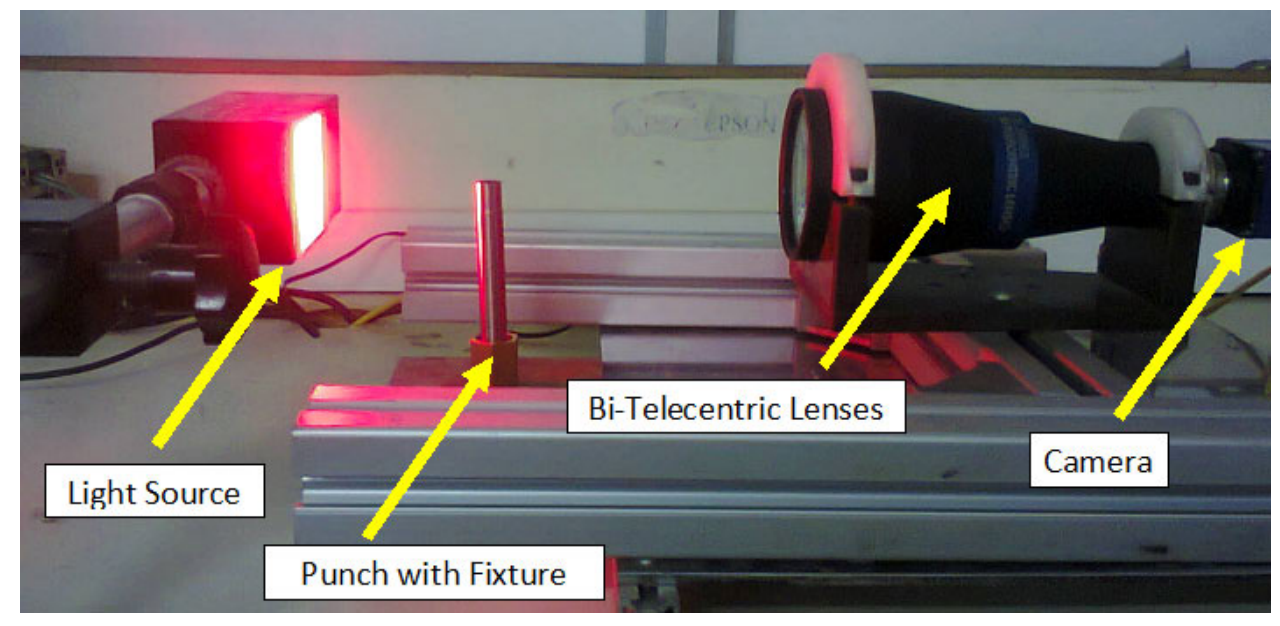

Fig. 6 Machine vision setup for capturing punch images

\subsubsection{Image processing}

The acquired images are subjected to image processing to extract the feature to describe the punch wear. The image processing technique involves image cropping, noise reduction, image thresholding, image cleaning, and feature extraction. The NI Vision Assistant software is used for processing the acquired images. A measurement program using the LabView software developed for the punch wear measurement is used to find wear on the punches from the acquired images. An image of a used punch tip acquired by the machine vision setup is shown in Fig. 7 and the graphical user interface of the LabView software showing wear in pixels at a predetermined distance from the punch edge is shown in Fig. 8. Edge detection is used in the processing of the images in the Labview software to assess wear progression in the punches as shown in Fig. 9. 

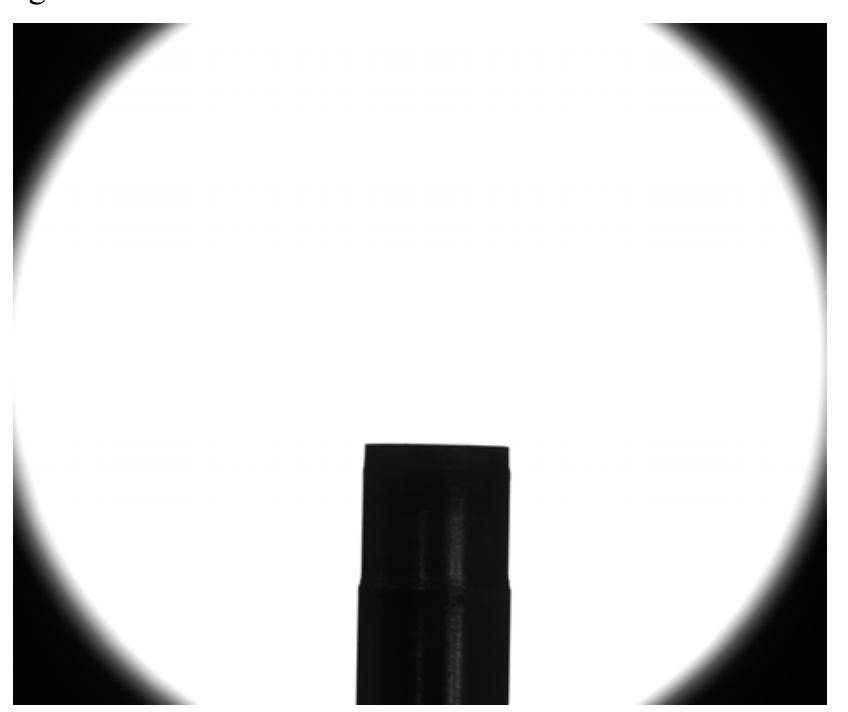

Fig. 7 Punch tip image captured by the machine vision module

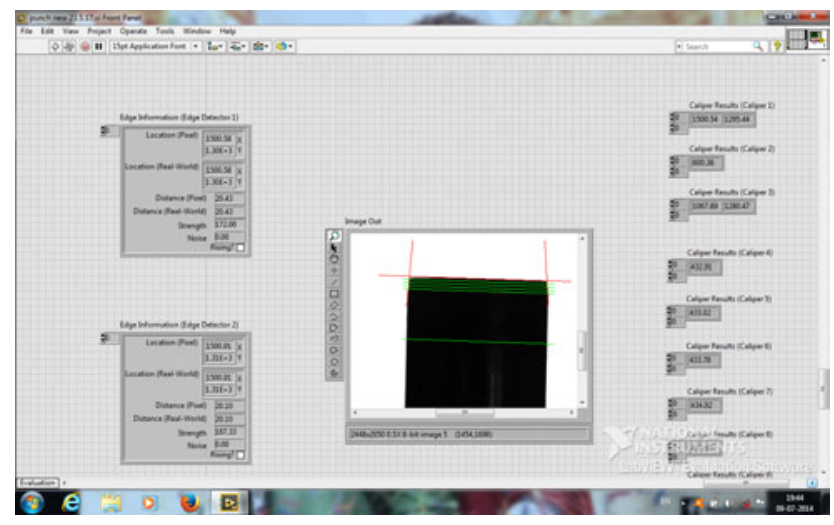

Fig. 8 Graphical user interface of the Labview software for processing images

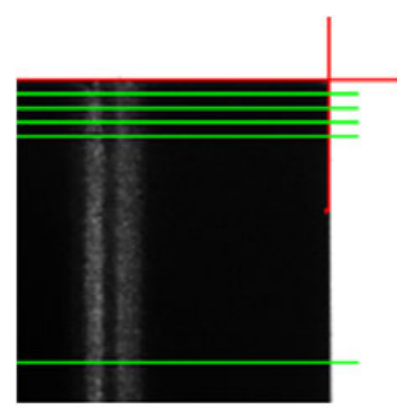

(a)

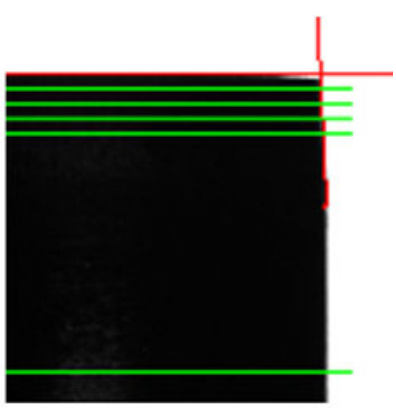

(b)

Fig. 9 Edge detection for assessment of wear progression in (a) unused punch tip and (b) used punch tip

\subsection{Pin-on-disk wear test}

The laboratory test using a pin-on-disk dry sliding wear testing machine was performed according to the ASTM standard (G99-05) using a computerized wear and friction monitor (DUCOM TR 201). This tribometer consists of a rotating disk on which a test pin is pressed with a known force. The tribometer is connected with a computer, by which the height loss of the pin and the frictional force at the interface of pin and disk are continuously recorded. Fig. 10 and Fig. 11 show the schematic and actual views of the pin-on-disk wear testing machine. 
During testing, pins made of D-3 steel subjected to different treatments as discussed earlier were kept stationary while the circular disk made of the hardened D-3 steel was rotated. Cylindrical pins required for the wear testing are made to $8 \mathrm{~mm}$ in diameter and 25 $\mathrm{mm}$ in length to suit the specifications of the tribometer. Also, a hardened D-3 steel disk of 80 $\mathrm{mm}$ in diameter and $8 \mathrm{~mm}$ thick was used as rotating member against which the pins were pressed with a known force.

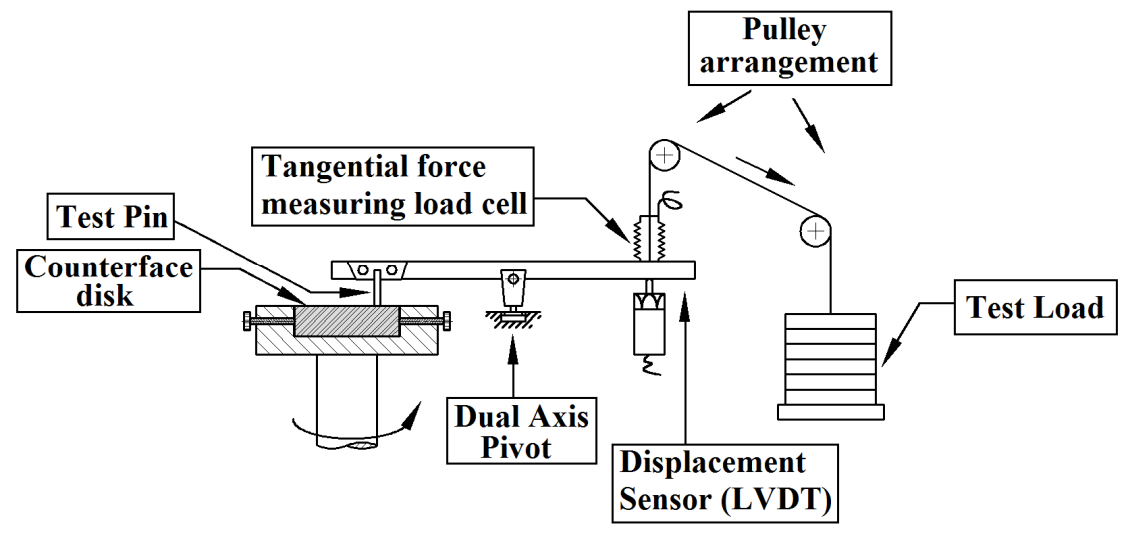

Fig. 10 Schematic diagram of pin-on-disk wear testing machine

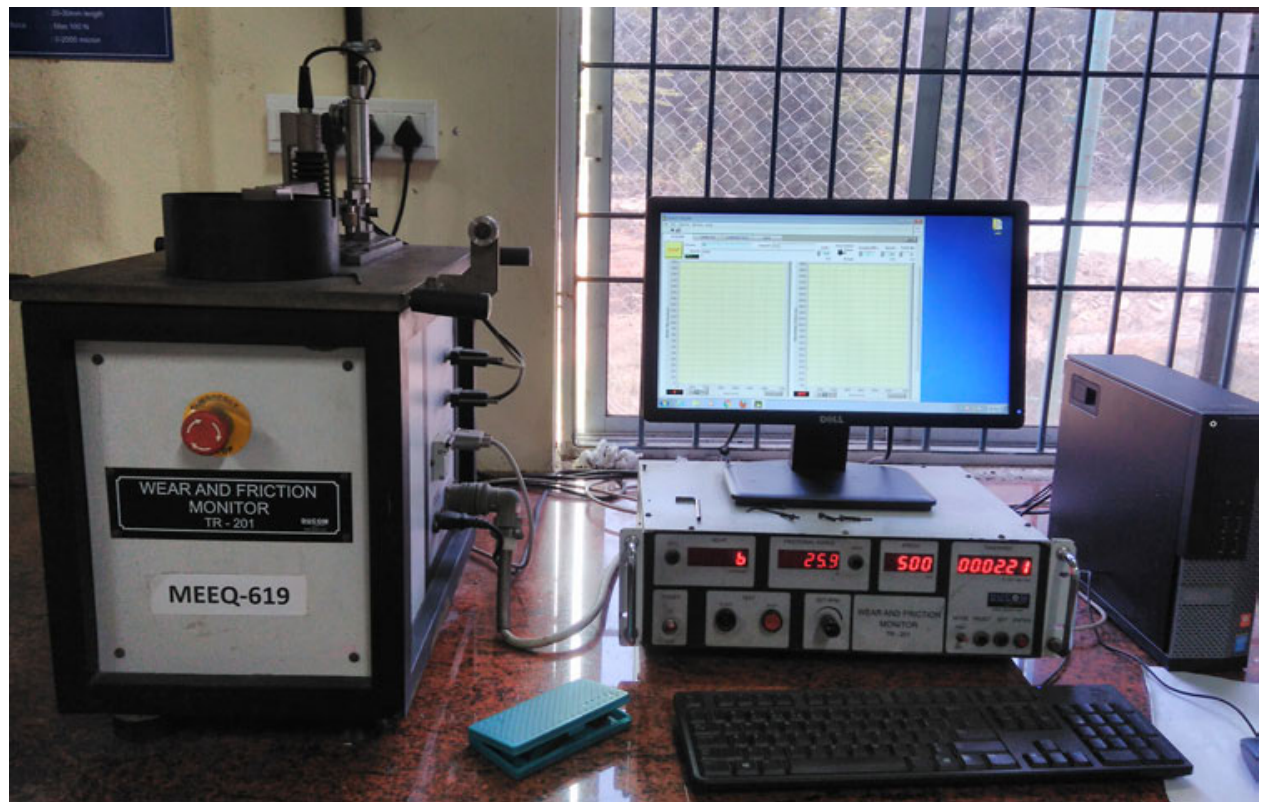

Fig. 11 Tribometer used for pin-on-disk wear test

Experiments were conducted by pressing the D-3 steel pins after four different treatments (HT, HC, HCT and HTC) against the hardened D-3 disk, using the pin-on-disk tribometer. The data acquisition system acquired the height loss of the pins and the frictional force when the test was conducted. A constant load of $50 \mathrm{~N}$ was applied on the pins against the disk rotating at a speed of $500 \mathrm{rpm}$ for a duration of 15 minutes in all the cases of four categories of samples. During the tests wear debris were not removed from the disk surface to simulate real life environment. 
Field and Laboratory Investigations

\section{Results and discussion}

\subsection{Hardness tests}

The results of the micro-hardness tests of the D-3 specimens after treatments are shown as equivalent HRC values in Fig. 12.

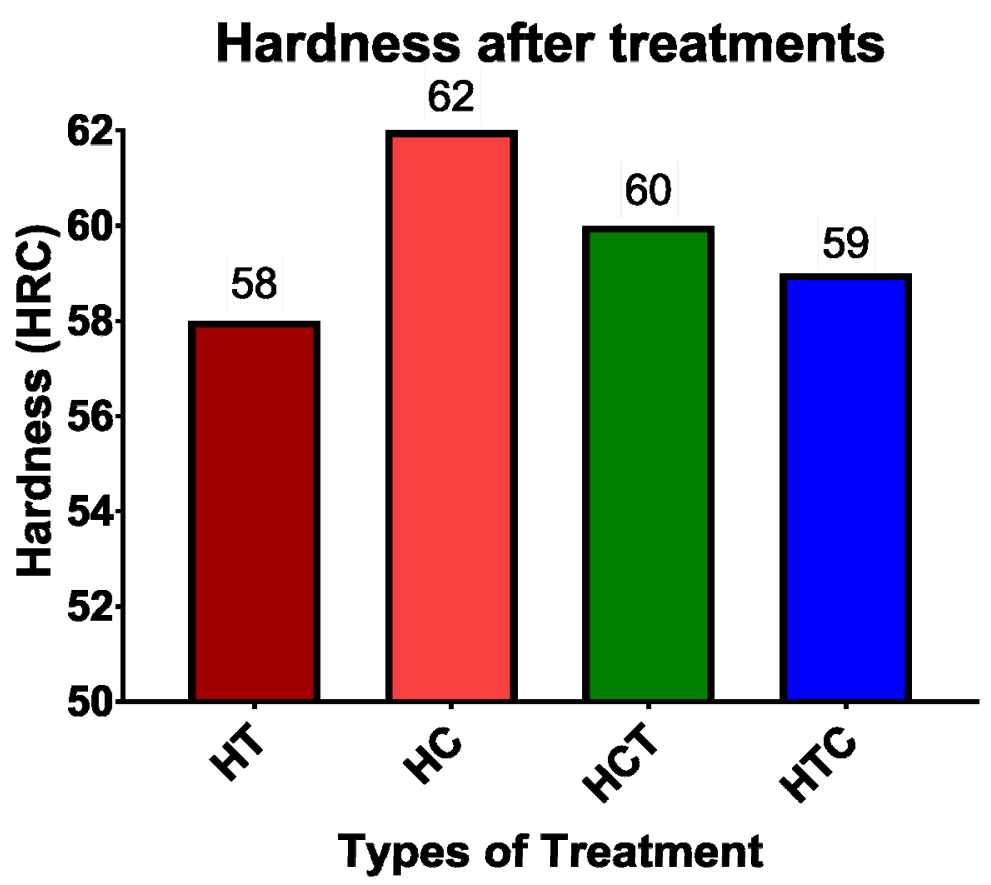

Fig. 12 Hardness values of specimens after treatments

The results show that the cryogenic treatment resulted in a considerable increase in hardness compared to the conventionally heat-treated (HT) specimens. The specimens of the HC group attained highest hardness due to the cryogenic treatment performed immediately after hardening. This is because of the elimination of austenite retained after hardening by converting it into martensite during cryogenic cooling. The results regarding hardness show that the cryogenic treatment (HCT) reduced hardness by $2 \mathrm{HRC}$ in comparison with the specimens in the $\mathrm{HC}$ group due to the transformation of the retained austenite into martensite, precipitation and uniform distribution of homogenized carbide. Reduction in hardness was also attributed to the reduction in tetragonality of the martensite's lattice structure during tempering which occurred immediately after hardening. The hardness of the HTC specimens is reduced by $1 \mathrm{HRC}$ in comparison with the HCT group due to the stabilization of austenite (retained after hardening) during tempering and the difficulty in transforming this stabilized austenite into martensite as cryogenic treatment was performed after tempering.

\subsection{Microstructural analysis}

The microstructural analysis carried out on the D-3 tool steel specimens revealed the effects of cryogenic treatment and tempering. The images acquired from the optical microscope are shown in Fig. 13. 


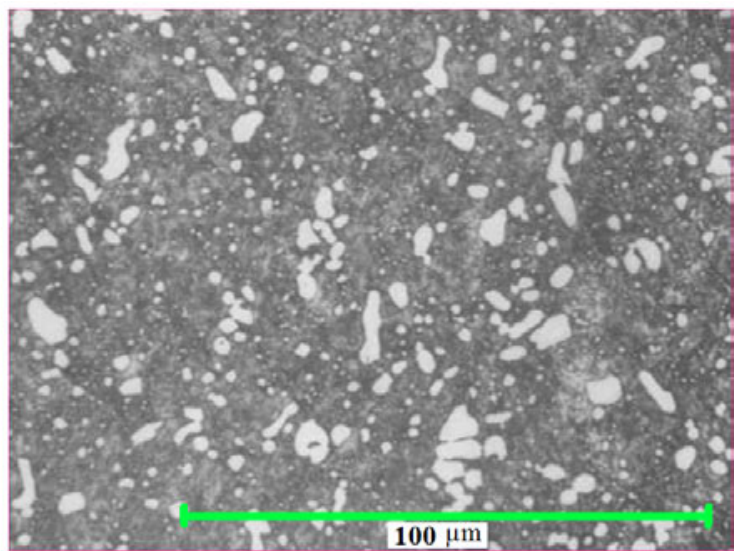

(a) HT

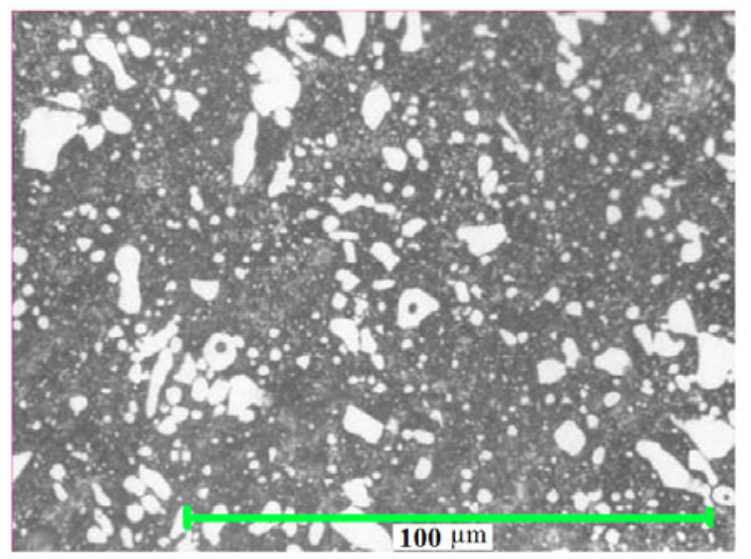

(c) HCT

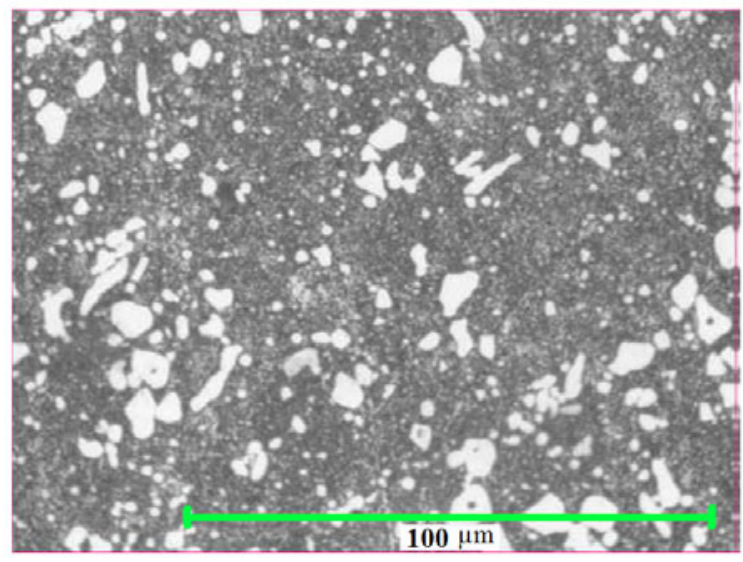

(b) $\mathrm{HC}$

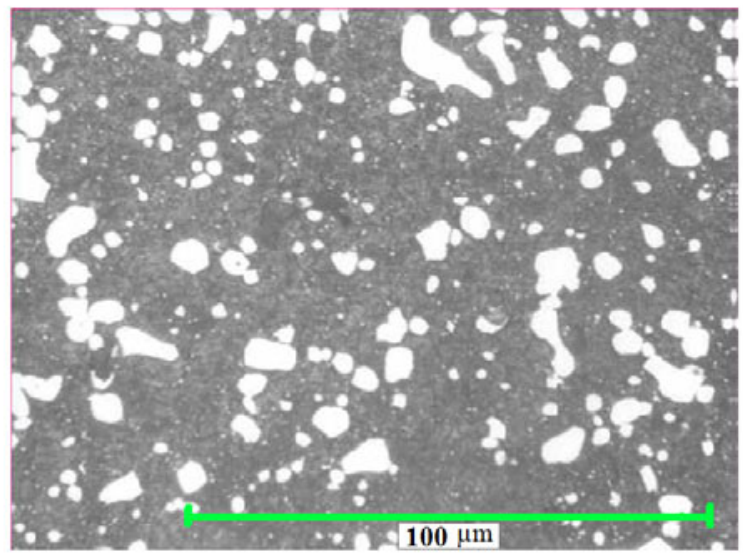

(d) HTC

Fig. 13 Optical micrographs of differently treated specimens at $100 \mu \mathrm{m}$ from the surface @ 1000X

The microstructure of the HT specimen (Fig. 13a) reveals uniformly distributed globular and nodular carbides in the tempered martensite matrix. The conventional hardening treatment results in a significant amount of untransformed austenite in the matrix which has undesirable effects on material properties. The microstructure of the simply cryogenically cooled (HC) specimen (Fig. 13b) exhibits pre-existing carbides in the matrix. However, the reduction in size of the globular carbides is observed in the HC specimen compared to that of the conventionally hardened (HT) specimen. Untempered martensite is present in the microstructure. Uniformly distributed carbides are present in the austenite matrix. The microstructure of the HCT sample (Fig. 13c) exhibits a significantly reduced carbide size compared to that of the HT specimen. Uniformly distributed globular and nodular carbides are present in the tempered matrix structure. The residual austenite presence is almost eliminated. The microstructure of the HTC specimen (Fig. 13d) in which case the tempering process was carried out immediately after hardening and subsequently the cryogenic cooling reveals globular carbides and carbides of irregular shape equally observed in the tempered martensite matrix with untempered martensite presence. Most of the retained austenite in the HT specimen is converted into martensite in the HC, HCT and HTC specimens, thereby increasing their hardness and improving wear resistance with respect to HT specimens. In addition, the increased carbide precipitation and increased carbide size seen in the microstructure of the HC, HCT and HTC specimens compared to the HT specimens are also responsible for improved wear resistance and hardness. Diminished grain boundaries and 
Field and Laboratory Investigations

dispersed carbides in the matrix also attribute to the increase in wear resistance when cryogenic treatment is applied.

SEM images of the specimens treated at $3500 \mathrm{X}$ magnification were also acquired to ensure the presence of fine carbides. The SEM images of the treated specimens are shown in Fig. 14.

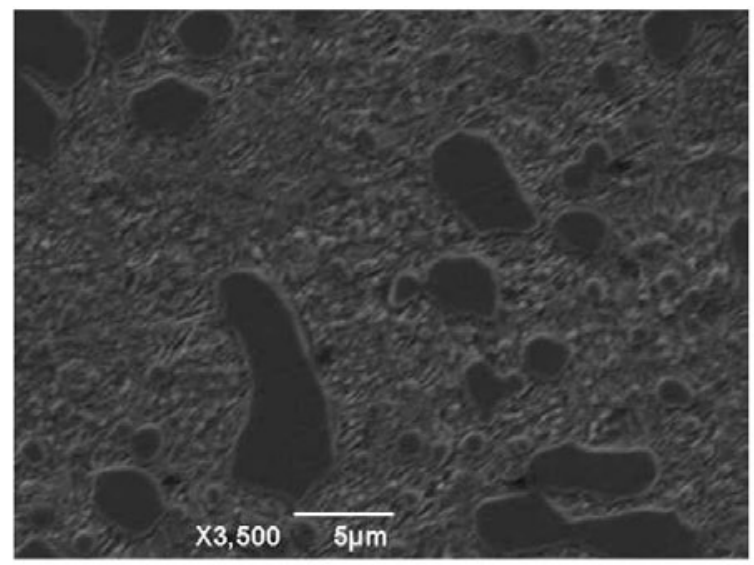

(a) HT

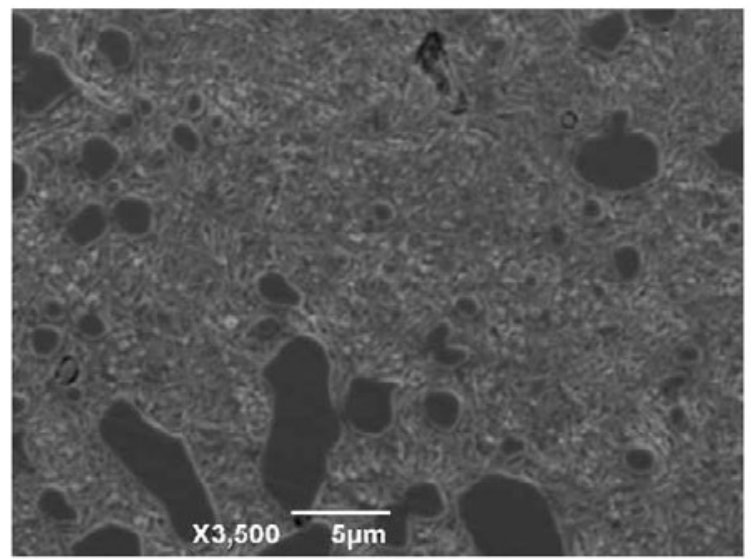

(c) $\mathrm{HCT}$

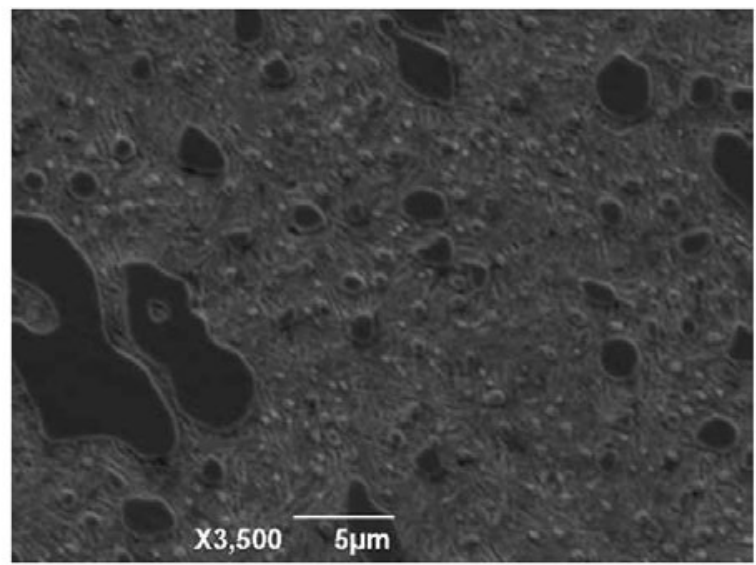

(b) $\mathrm{HC}$

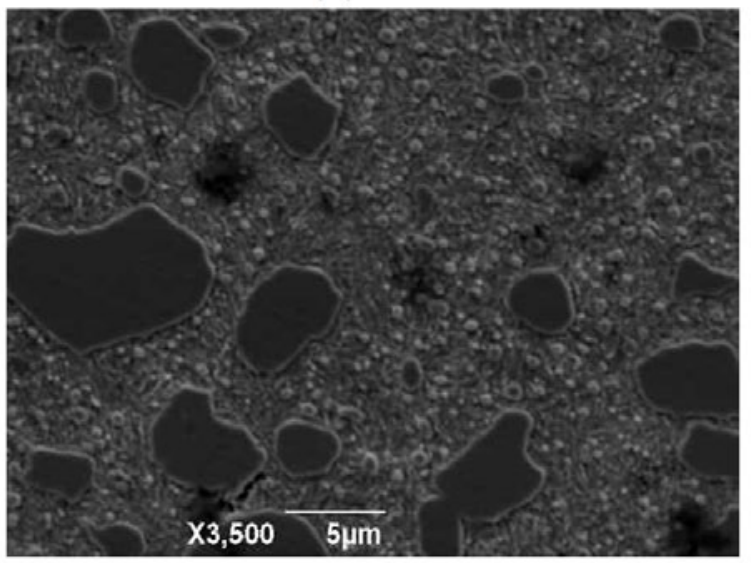

(d) HTC

Fig. 14 SEM micrographs of differently treated D-3 specimens @3500X

The SEM micrograph of the HT specimen (Fig. 14a) exhibits uniformly distributed large elongated dendritic primary carbides and nearly spherical larger and smaller secondary carbides in the tempered martensitic matrix with presence of undissolved carbides. The SEM micrograph of the $\mathrm{HC}$ specimen (Fig. 14b) shows undissolved and fine carbides present in the martensite matrix. Untempered martensite is present in the structure. The SEM micrograph of the HCT sample (Fig. 14c) exhibits undissolved carbides in the tempered martensitic matrix which became unprecipitated and are considered as primary carbides. Hence no retained austenite is present. The secondary carbides in the microstructure are of refined sizes, increased population and uniformly distributed in comparison with the HT specimen. The SEM micrograph of the HTC sample (Fig. 14d) shows undissolved carbides in the tempered martensite matrix and precipitated fine carbides. From the analysis of the SEM images it is observed that the cryogenic treatment facilitated the refinement, increased population density and uniform distribution of the secondary carbides in the martensitic matrix structure. 
3.3 Wear analysis by machine vision system

The piercing punches undergo sliding wear due to the sliding action between the punches and the pierced part. The progression of wear of the punches with the number of strokes was estimated at a distance of $0.5 \mathrm{~mm}$ from the punch edge using machine vision system captured images by applying the image processing technique. The results of this examination are shown in Fig. 15 presenting the average of five measurements taken at different punch positions. As expected, wear increases with the increase in the number of strokes. The wear progressions of conventionally and cryogenically treated punches are evaluated in the field tests after up to 28,000 strokes. The results indicate that the cryogenic treatment enhanced wear resistance of the piercing punches.

\section{Wear on piercing punches at a distance} of $0.5 \mathrm{~mm}$ from punch edges

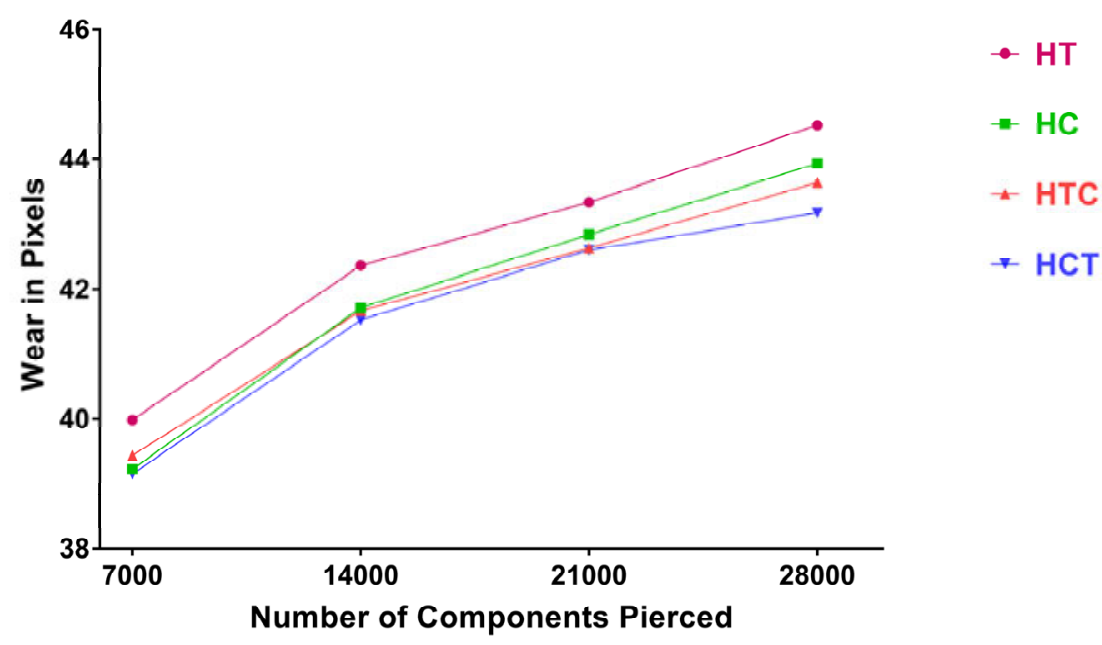

Fig. 15 Measurement of wear progression from the punch edge as a function of number of strokes

The wear rate of the HT punches is the highest because of the presence of retained austenite in its microstructure. The wear rate of the $\mathrm{HC}$ punches is lower compared to that of the HT punches due to the conversion of retained austenite into martensite during the cryogenic treatment. Tempering before the cryogenic treatment in the HTC punches stabilizes the retained austenite after hardening making it difficult to be transformed during the cryogenic treatment. Hence the wear rate of the HTC punches is slightly higher than that of the HCT punches. Tempering after the cryogenic treatment in the HCT punches results in the precipitation of carbide particles which leads to improved toughness and wear resistance. Hence the HCT punches show the lowest wear rate.

\subsection{Wear analysis by applying the pin-on-disk method}

The pin-on-disk wear test conducted on the cryogenically treated D-3 steel specimens shows a remarkable drop in wear as evident from the plots shown in Fig. 16 and Fig. 17. The plots in Fig. 18 reveal the frictional force of the conventionally hardened and the cryogenically treated specimens for a constant load of $50 \mathrm{~N}$ and a constant speed of $500 \mathrm{rpm}$. 
Wear measured during pin-on-disk wear test

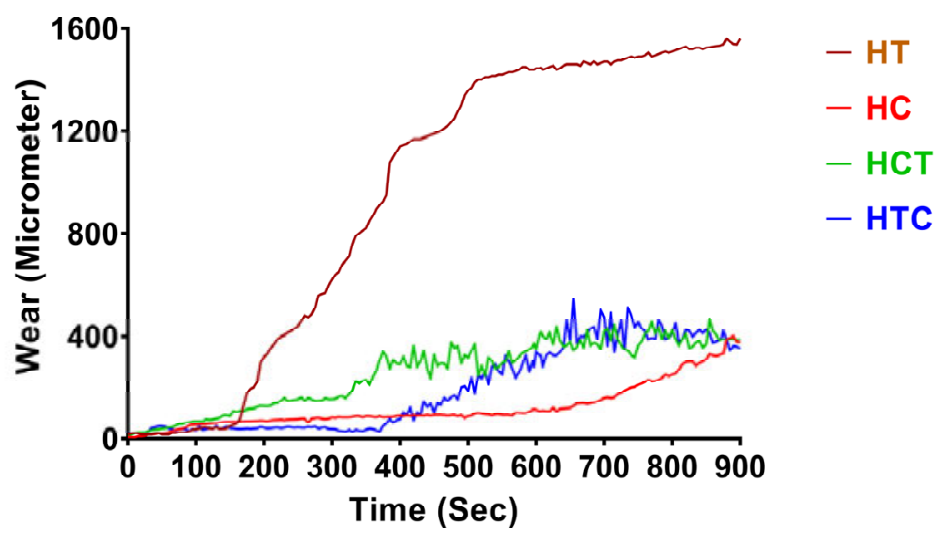

Fig. 16 Wear behaviour of D-3 specimens under a normal load of $50 \mathrm{~N}$, speed of $500 \mathrm{rpm}$ and duration of 15 minutes

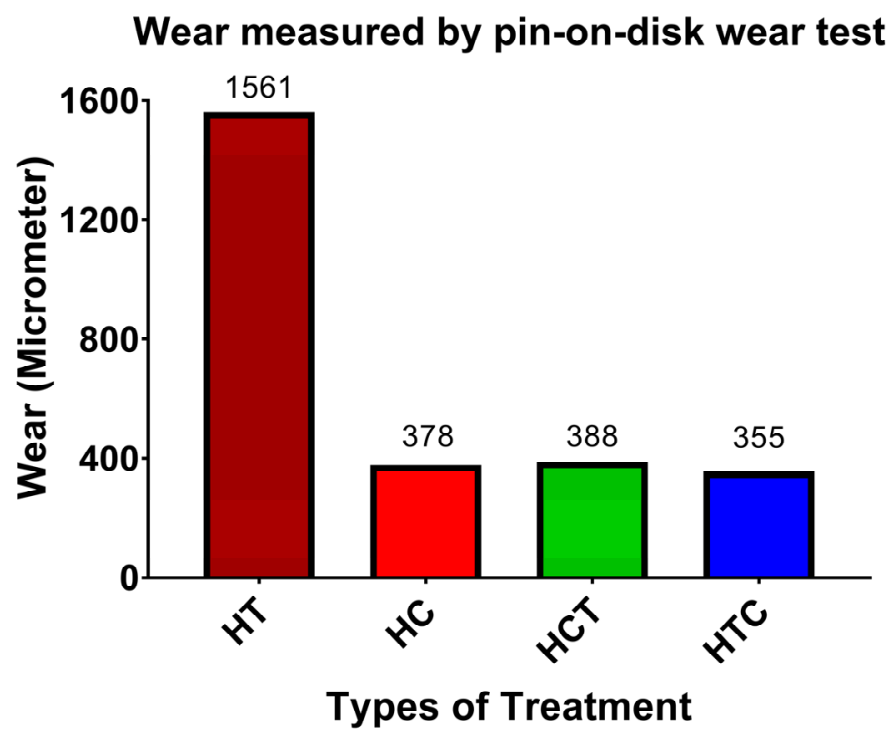

Fig. 17 Comparison of wear measured by pin-on-disk tribometer

Frictional force measured during pin-on-disk wear test

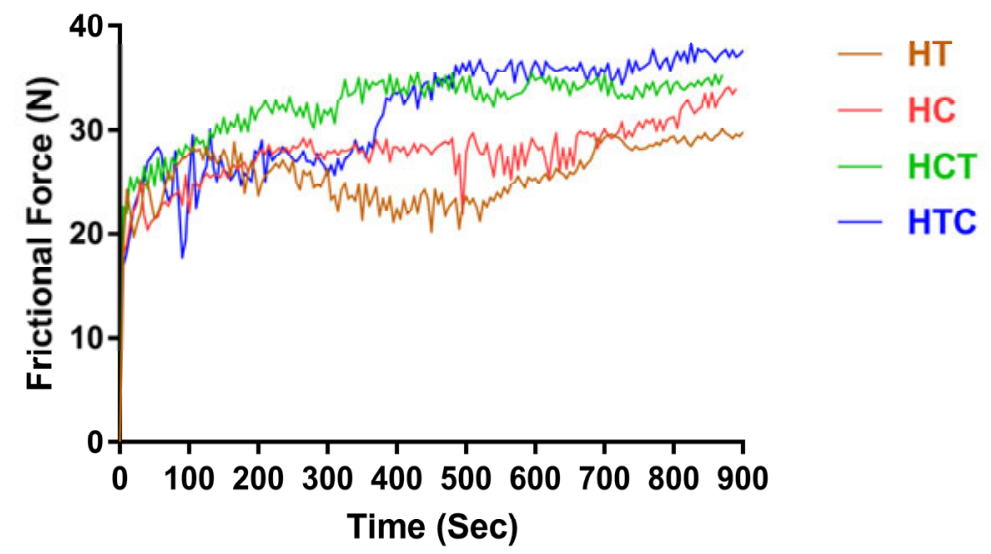

Fig. 18 Plot of the friction force during pin-on-disk wear test 
The lowest wear is shown in the HCT specimens as a result of precipitation of fine carbides during the cryogenic treatment. Fine carbides precipitation imparts wear resistance to the material during sliding wear. Wear of the HT specimens is the highest. High wear of the HT specimens could be attributed to primarily coarse carbides and retained austenite. The cryogenically treated samples wear out slowly when compared to the conventionally hardened samples confirming the enhancement of wear resistance by nearly 4 times. The comparison of the frictional force between the conventionally hardened and the cryogenically treated samples reveals better performance of the material that underwent cryogenic treatment.

\section{Conclusions} are drawn:

In the present paper based on shop floor and laboratory tests the following conclusions

- In general, cryogenic treatment increases hardness of D-3 steel specimens more than conventional heat treatment. The hardness value of the samples gradually increased in the following ascending order: HT, HTC, HCT, HC.

- The microstructural studies on the cryogenically treated specimens reveal that the untransformed soft austenite retained after quenching is transformed into hard martensite and carbide precipitation occurs during cryogenic treatment. These changes will enhance wear resistance and toughness of D-3 steel specimens and increase their life.

- The results of the shop floor experiments conducted with a machine vision system on the punches used for the production of chain parts confirm that the cryogenic treatment enhances punch life considerably. The punch life increases in the following ascending order: HT, HC, HTC, HCT.

- The results of the pin-on-disk wear experiments show that the wear rate of the HT specimen is the highest, approximately 4 times higher than that of the cryogenically treated specimens. Although there is no much difference in wear rates of the HC, HCT and HTC specimens the wear rate of the HCT specimen uniformly increases in comparison with the $\mathrm{HC}$ and HTC specimens. In the case of the HC specimen this may be because of the absence of tempering and in the case of the HTC specimen, because tempering was carried out before the cryogenic treatment. punches.

These results suggest that the cryogenic treatment increased wear life of D-3 piercing

\section{REFERENCES}

[1] K. Lawanwong, N. Pornputsiri, G. Luangsopapun, An investigation of adhesion wear behavior of tool steel on blanking die, IPCSIT, 2011; 15: 25-30

[2] PVD. Marcondes, AM. Eto, PAC. Beltrăo, PC. Borges, A smart stamping tool for punching and broaching combination., Journal of Materials Processing Technology, 2008, 206:184-193 https://doi.org/10.1016/j.jmatprotec.2007.12.005

[3] Onur Çavuşoğlu, Hakan Gürün, The relationship of burr height and blanking force With clearance in the blanking process of aa5754 Aluminium alloy, Transactions of FAMENA, 2017; 41(1):55-62; https://doi.org/10.21278/TOF.41105

[4] H. Makich, L. Carpentier, G. Monteil, X. Roizard, J. Chambert, P. Picart, Metrology of the burr amount-correlation with blanking operation parameters (blanked material-wear of the punch), Int $J$ Mater Form (C) Springer/ESAFORM, Suppl., 2008; 1:1243-1246

[5] W.B.Lee, C.F.Cheung, W.M.Chiu, L.K.Chan, Automatic supervision of blanking tool wear using Pattern recognition analysis, International Journal of Machine Tools and Manufacture, 1997; 37:1079-1095 
Enhancement of Wear Resistance of D-3 Piercing

Punches by Applying Cryogenic Treatment:

Field and Laboratory Investigations

[6] J.J.Hernández, P.Franco, M.Estrems, F.Faura, Modelling and experimental analysis of the effects of tool wear on form errors in stainless steel blanking, Journal of Materials Processing Technology, 2006; 180:143-150; https://doi.org/10.1016/j.jmatprotec.2006.05.015

[7] Ridha Hambli, Prediction of burr height formation in blanking processes using neural network, International Journal of Mechanical Sciences, 2002; 44:2089-2102; https://doi.org/10.1016/S00207403(02)00168-6

[8] S.Y Luo, Effect of the geometry and the surface treatment of punching tools on the tool life and wear conditions in the piercing of thick steel plate, Journal of Materials Processing Technology, 1999; 88:122133; https://doi.org/10.1016/S0924-0136(98)00375-6

[9] Dae-Cheol Ko, Dong-Hwan Kim, Byung-Min Kim, Finite element analysis for the wear of Ti-N coated punch in the piercing process, Wear, 2002; 252:859-869; https://doi.org/10.1016/S0043-1648(02)00032-7

[10] Ridha Hambli, Blanking tool wear modeling using the finite element method, International Journal of Machine Tools and Manufacture, 2001; 41:1815-1829; https://doi.org/10.1016/S0890-6955(01)00024-4

[11] F. Faura, J. Lopez, MA. Sabastian, C. Luis, Blanking of stainless steel: tool life equation model, Advanced Technology of Plasticity, 1996; 2:655-663

[12] T. Maeda, I. Aoki, The study on wear of blanking tool for 18-8 stainless steel and bainite hardened steel strips, J. of the Faculty of Engineering, University of Tokyo, 1974; 32:443-475

[13] S.Y. Luo, Studies on the wear conditions and the sheared edges in punching, Wear, 1997; 208:81-90; https://doi.org/10.1016/S0043-1648(96)07439-X

[14] D.C.D. Oguamanam, H. Raafat, S.M. Taboun, A machine vision system for wear monitoring and breakage detection of single-point cutting tools, Computers \& Industrial Engineering, 1994; 26:575-598; https://doi.org/10.1016/0360-8352(94)90052-3

[15] S. Dutta, S.K. Pal, S. Mukhopadhyay, R. Sen, Application of digital image processing in tool condition monitoring: A review, CIRP Journal of Manufacturing Science and Technology, 2013; 6:212-232; https://doi.org/10.1016/j.cirpj.2013.02.005

[16] Myo Hla Myint, J.Y.H Fuh, Y.S Wong, Z.D Chen, C.M Choy, Evaluation of wear mechanisms of Y-TZP and tungsten carbide punches, Journal of Materials Processing Technology, 2003; 140:460-464; https://doi.org/10.1016/S0924-0136(03)00762-3

[17] X.T. Zeng, S. Zhang, T. Muramatsu, Comparison of three advanced hard coatings for stamping applications, Surface and Coatings Technology, 2000; 127:38-42; https://doi.org/10.1016/S02578972(99)00668-4

[18] Daniel Tobola, Witold Brostow, Kazimierz Czechowski, Piotr Rusek, Improvement of wear resistance of some cold working tool steels, Wear, 2017; 382 - 383:29-39; https://doi.org/10.1016/j.wear.2017.03.023

[19] Jerry Dwyer, 12 ways to boost punch life, STAMPING Journal, http://www.thefabricator.com/publication/sj, August 2007

[20] Liina LIND, Priidu PEETSALU, Fjodor SERGEJEV, Wear of Different PVD Coatings at Industrial Fineblanking Field Tests, Materials Science, 2015; 21:343-348; http://dx.doi.org/10.5755/j01.ms.21.3.7249

[21] D. Das, K.K. Ray and A.K. Dutta, Influence of temperature of sub-zero treatments on the wear behaviour of die steel, Wear, 2009; 267:1361-1370; https://doi.org/10.1016/j.wear.2008.11.029

[22] Adem Çiçek, Turgay Kıvak, Ilyas Uygur, Ergün Ekici, Yakup Turgut, Performance of cryogenically treated M35 HSS drills in drilling of austenitic stainless steels, The International Journal of Advanced Manufacturing Technology, 2012; 60:65-73; https://doi.org/10.1007/s00170-011-3616-8

[23] Arslan Y, Uygur I, Jazdzewska A, The effect of cryogenic treatment on microstructure and mechanical response of AISI D3 tool steel punches, Journal of Manufacturing Science and Engineering, 2015; 137(3): 6 pages; http://dx.doi.org/10.1115/1.4029567

[24] K.K. Ray, D. Das, Improved wear resistance of steels by cryotreatment: the current state of understanding, Materials Science and Technology, 2017; 33:340-354; http://dx.doi.org/10.1080/02670836.2016.1206292

[25] Shaohong Li, Yinzi Xie, Xiaochun Wu, Hardness and toughness investigations of deep cryogenic treated cold work die steel, Cryogenics, 2010; 50:89-92; https://doi.org/10.1016/j.cryogenics.2009.12.005

[26] A. Molinari, M. Pellizzari, S. Gialanella, G. Straffelini, KH. Stiasny, Effect of deep cryogenic treatment on the mechanical properties of tool steel, Journal of Materials Processing Technology, 2001; 118:350355; https://doi.org/10.1016/S0924-0136(01)00973-6 
[27] Fla'vio J. da Silva, Sine'sio D. Franco, A' lisson R. Machado, Emmanuel O. Ezugwu and Ant`onio M. Souza Jr., Performance of cryogenically treated HSS tools, Wear, 2006; 261:674-685; https://doi.org/10.1016/j.wear.2006.01.017

[28] A.Y.L. Yong, K.H.W. Seah and M. Rahman, Performance evaluation of cryogenically treated tungsten carbide tools in turning, International Journal of Machine Tools \& Manufacture, 2006; 46:2051-2056; https://doi.org/10.1016/j.ijmachtools.2006.01.002

[29] V. Firouzdor, E. Nejati and F. Khomamizadeh, Effect of deep cryogenic treatment on wear resistance and tool life of M2 HSS drill, Journal of materials processing technology, 2008; 206:467-472; https://doi.org/10.1016/j.jmatprotec.2007.12.072

[30] N.B. Dhokey and S. Nirbhavne, Dry sliding wear of cryotreated multiple tempered D-3 tool steel, Journal of materials processing technology, 2009; 209:1484-1490;

https://doi.org/10.1016/j.jmatprotec.2008.03.069

Submitted: $\quad 23.11 .2017$

Accepted: $\quad$ 15.3.2018
Karuppan Subramani Sekar

Lecturer,

Murugappa Polytechnic College,

S.M.Nagar, Chennai - 600 062,

Tamilnadu, India

Email: emailsekarks@gmail.com

Suruli Nagarajan Murugesan

Professor,

Rajalakshmi Engineering College,

Thandalam, Chennai - 602 105,

Tamilnadu, India 